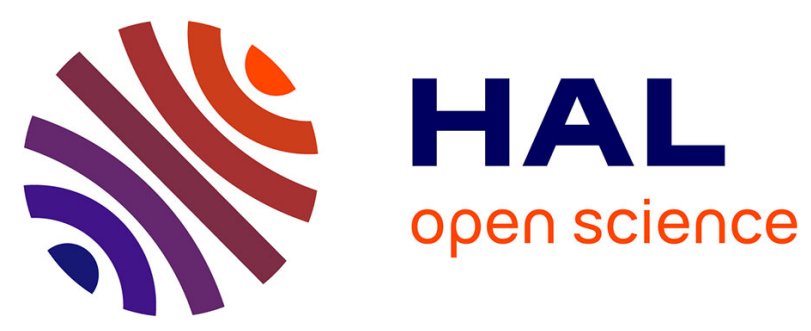

\title{
Pilotage des systèmes manufacturiers via une analyse multicritère intégrant Produit, Ressource et Ordre. Application au pilotage en flux tiré d'un Job Shop
} Patrick Pujo, Fouzia Ounnar, Pascal Blanc

\section{- To cite this version:}

Patrick Pujo, Fouzia Ounnar, Pascal Blanc. Pilotage des systèmes manufacturiers via une analyse multicritère intégrant Produit, Ressource et Ordre. Application au pilotage en flux tiré d'un Job Shop. Journal Européen des Systèmes Automatisés (JESA), 2009, 43 (3/4), pp.435-462. 10.3166/JESA.43.435-462 . hal-01212610

\author{
HAL Id: hal-01212610 \\ https://hal.science/hal-01212610
}

Submitted on 8 Nov 2015

HAL is a multi-disciplinary open access archive for the deposit and dissemination of scientific research documents, whether they are published or not. The documents may come from teaching and research institutions in France or abroad, or from public or private research centers.
L'archive ouverte pluridisciplinaire HAL, est destinée au dépôt et à la diffusion de documents scientifiques de niveau recherche, publiés ou non, émanant des établissements d'enseignement et de recherche français ou étrangers, des laboratoires publics ou privés. 


\title{
Pilotage des systèmes manufacturiers via une analyse multicritère intégrant Produit, Ressource et Ordre
}

\author{
Application au pilotage en flux tiré d'un Job Shop \\ Patrick PUJO, Fouzia OUNNAR, Pascal BLANC \\ Laboratoire des Sciences de l'Information et des Systèmes \\ Aix-Marseille Université \\ Avenue Escadrille Normandie Niemen, F-13397 Marseille cedex 20 \\ \{patrick.pujo ; fouzia.ounnar ; pascal.blanc\}@lsis.org
}

\begin{abstract}
RÉSUMÉ. Les conditions de plus en plus exigeantes de la production industrielle imposent de rechercher de nouvelles techniques de pilotage d'atelier. Nous présentons ici une manière radicalement novatrice pour organiser le pilotage, reposant sur l'approche holonique, l'isoarchie et l'analyse multicritère. Après avoir abordé les différents concepts sous-tendant cette approche, nous détaillons les mécanismes de décision multicritère utilisés et la façon de la mettre en cuvre et de l'instrumenter. Les premiers résultats obtenus par simulation via Arena et un module de calcul multicritère AHP sont ensuite présentés et montrent les excellentes performances obtenues. Enfin, nous détaillerons la plateforme de test que nous venons d'achever. L'objectif est de pouvoir explorer et analyser plus facilement les performances de cette nouvelle approche du pilotage d'atelier.
\end{abstract}

ABSTRACT. The more and more requirements of the industrial production force to seek new techniques of workshop control. We present in this paper a radically innovative manner to organize control, based on the holonic approach, the isoarchy structure and the multicriteria analysis. After having approached the various concepts related to this approach, we detail the used multicriteria decision mechanisms and the way of implementing the proposed approach and of instrumenting it. The first results obtained by simulation via Arena and by an AHP multicriteria calculation module are then presented and show the excellent obtained performances. Lastly, we will detail the test platform which we have just completed. The objective is to be able to explore and analyze more easily the performances of this new approach of the workshop control.

MOTS-CLÉS : Pilotage, Holarchie, Isoarchie, Analytic Hierarchy Process, flux tiré, Job Shop.

KEYWORDS: Workshop control, Holarchy, Isoarchy, Analytic Hierarchy Process, Pull control, Job Shop.

\section{Introduction}

L'évolution des conditions de concurrence dans le monde de la production industrielle se fait au détriment des entreprises devant supporter un coût du travail élevé. Si certaines d'entre elles choisissent d'externaliser ou de délocaliser, d'autres choisissent au contraire de rechercher de nouvelles solutions pour augmenter la productivité. Toutefois, l'ensemble des méthodes et des technologies mises en œuvre dans l'industrie durant ces dernières décennies semblent avoir atteint leurs limites et les entreprises aimeraient que leur soient proposées de nouvelles solutions leur permettant des gains substantiels de productivité.

Parallèlement, le monde scientifique a proposé depuis une vingtaine d'années de nombreuses idées innovantes, principalement basées sur des architectures hétérarchiques de pilotage. Malheureusement, les applications sont souvent restées au stade d'exemples élémentaires ou de démonstrateurs canoniques ou académiques.

Après avoir présenté dans un premier temps l'évolution des concepts fondant le fonctionnement des systèmes de pilotage pour systèmes manufacturiers, nous rappelons les solutions les plus connues proposées par le monde scientifique et plus particulièrement l'architecture holonique PROSA. Nous exposons ensuite notre propre approche, PROSIS, développée dans un cadre holonique en filiation de PROSA, mais présentant un cadre exclusivement isoarchique et permettant une prise de décision multicritère. Puis, nous détaillons ces mécanismes de décision, en explicitant les motivations qui les sous tendent et la structure de mise en œuvre via l'algorithme AHP (Analytic Hierarchy Process). Ensuite, nous présentons l'application de cette approche à une situation industrielle réelle, avec des premiers résultats obtenus en simulation sur un démonstrateur ad hoc. Cette expérimentation a montré une nette diminution des stocks d'encours et des temps d'attente, donc une réduction des coûts de production associés. Enfin, nous décrivons la plateforme de simulation que nous avons développée pour continuer l'étude de l'approche PROSIS. Cette plateforme, qui met en œuvre les concepts liés à PROSIS, permet soit un usage en simulation - émulation, soit le pilotage réel d'une installation. Cet outil polyvalent, c'est-à-dire capable de s'adapter à tout type d'atelier fonctionnant en Job Shop, va nous permettre d'explorer les performances atteignables par l'appel à un mécanisme de décision multicritère et de comprendre les conditions de paramétrage de cet algorithme. 


\section{Pilotage holonique isoarchique}

Dans ce paragraphe, nous montrons comment ont évolué les méthodes de pilotage en production manufacturière.

En particulier, nous rappelons les circonstances ayant engendrées l'apparition de l'approche du pilotage par le produit et nous expliquons pourquoi cette approche doit être enrichie par un pilotage multicritère s'appuyant sur le paradigme holonique et une architecture isoarchique.

\subsection{Evolution du pilotage des systèmes de production}

Les systèmes de pilotage sont en perpétuelle évolution. Nous présentons ici comment, à partir des approches conventionnelles du pilotage, se développent actuellement de nouvelles approches et comment nous nous singularisons par notre approche.

\subsubsection{Approches conventionnelles du pilotage}

Dans un atelier, le pilotage opérationnel doit indiquer de manière précise les actions à conduire à court terme, voire même en temps réel, pour pouvoir obtenir un fonctionnement efficace et efficient du système de production. En d'autres termes, il s'agit d'indiquer, selon l'Hexamètre de Quintilien, QUI fait QUOI, QUAND, OU et COMMENT, en respectant d'une part les contraintes définies aux niveaux décisionnels supérieurs et d'autre part celles inhérentes au système de production et à son environnement. Dans la quasi-totalité des cas, le QUI est associé au OU et le COMMENT est prédéfini pour chaque QUOI. L'organisation des tâches nécessite de définir un sous-ensemble du produit cartésien $\{\mathrm{QUOI}\} \times\{\mathrm{OU}\}$ x $\{\mathrm{QUAND}\}$ pour l'ensemble des tâches à réaliser. Cette activité d'organisation est générée par une planification menée hors ligne et de manière précise, conduisant à un ordonnancement du fonctionnement de l'atelier : c'est du pilotage prévisionnel (Pujo and Kieffer, 2002).

Lors de la mise en œuvre effective des tâches dans l'atelier apparaissent des évènements contingents qui viennent perturber ce plan initial. Le rôle du pilotage est alors de trouver en ligne une solution permettant à l'atelier de continuer à fonctionner en conservant des performances acceptables. Cet aspect opérationnel du pilotage est de plus en plus souvent réalisé par un MES (Manufacturing Execution System), sur la base d'un ré-ordonnancement des tâches sur les ressources ad hoc.

\subsubsection{Pilotage par le Produit}

Depuis une décennie, cette approche conventionnelle est remise en cause par quelques équipes de recherche : abandon de la recherche de l'optimisation d'un planning prévisionnel qui serait de toute façon remis en cause par l'apparition contingente d'évènements perturbateurs, prise en compte du rôle fonctionnel et participation des entités en interaction pour la recherche d'une solution de pilotage... Les solutions proposées s'appuient généralement sur une architecture de pilotage hétérarchique (Pujo et Brun Picard, 2001; Trentesaux, 2007). Parmi les nombreuses idées qui ont été proposées, nous pouvons évoquer différents travaux menés à Nancy et à Marseille. D'une part, (Chaxel et al, 1999) ont mis en évidence le rôle particulier qui peut être confié au produit, objet nomade disposant de son propre système d'information et sa propre capacité de décision. (Charpentier et al., 2001) ont proposé une formalisation matricielle des flux d'information entre produits et ressources au sein d'une hétérarchie. D'autre part, (Broissin, 1999) a montré l'intérêt de la prise en compte technique du produit dans la génération de tâches adaptatives et son rôle dans le choix dynamique et temps réel des équipements exécutant ces tâches. (Pujo et Brun Picard, 2001) proposent les concepts d'auto ordonnancement piloté par le produit ou par génération locale et contextuelle de tâches orientées métier.

Depuis quelques mois, une communauté scientifique s'est structurée autour des systèmes contrôlés par le produit (Bajic et al., 2007), dans le cadre d'un projet exploratoire du GDR MACS. Cette communauté s'intéresse à la maîtrise de l'interaction entre le procédé de fabrication et le produit, en intégrant dans la boucle cybernétique de nouvelles technologies telles que les communications sans fil (Wireless Sensors Network) et le RFID (Radio Frequency Identification). Ces technologies doivent permettre de doter le produit de capacités de mémorisation, de calcul et de communication : il devient alors «actif» au sein du système de production qui le traite. Ce produit « actif » peut être doté de moyens permettant de capter les variations de son environnement, de prendre des décisions et donc d'interagir pleinement avec son environnement (ressources constituant le procédé, autres produits, opérateurs humains...).

Ce projet exploratoire résulte de l'intérêt croissant de la communauté scientifique pour le contrôle par le produit (Morel et al., 2007). Nombreux sont les chercheurs qui voient dans ces nouvelles capacités l'opportunité d'améliorer largement les performances des différents aspects du pilotage des systèmes de production (McFarlane et al., 2002) (Gouyon, 2004) (Pétin et al., 2007) (Panetto et al., 2007). Cette voie est également préconisée par le Comité d'Experts en Productique du CNRS, dans son analyse prospective (Bourrières et al, 2007).

\subsubsection{Pilotage multi points de vue}

Toutefois, ce même comité fait état de la nécessité de "faire inter opérer des objets de nature différente en explorant les possibilités d'homogénéiser leurs capacités respectives d'interactions tout en maîtrisant l'émergence des propriétés issues de ces interactions multiples » et évoque le paradigme holonique, qui ne se réduit pas dans le domaine des HMS (Holonic Manufacturing System) (Mathews, 1995) à une vision uniquement orientée produit. Nous nous associons pleinement à cette vision de l'évolution des systèmes de pilotage.

A notre avis, le contrôle par le produit des systèmes de production n'est pas suffisant. En effet, le produit en tant que tel n'est pas porteur de toutes les contraintes de fonctionnement et de toutes les informations associées qui permettraient de prendre des 
décisions de pilotage optimales, ou au moins satisfaisantes. Cette position est largement confortée par les travaux de la communauté IMS (Intelligent Manufacturing System), qui ont permis d'identifier les différents types d'entités en interaction dans un système de production manufacturière. Chacune de ces entités amène son propre jeu de données et ses propres contraintes, constituant ainsi un point de vue. C'est la prise en compte de l'ensemble des données et des contraintes, parfois contradictoires, qui peut engendrer la meilleure solution possible, à un instant donné. C'est pourquoi nous prônons un pilotage intégrant de multiples points de vue et issu de différents types d'entités en interaction. Ceci est conceptuellement en accord avec les résultats issus des HMS (Holonic Manufacturing Systems).

\subsection{Architecture holonique du pilotage}

\subsubsection{Paradigme holonique}

Le paradigme holonique a été initialement imaginé par (Kostler, 1967) pour permettre la modélisation de systèmes sociaux complexes. Dans de tels systèmes, une entité (un holon) est tout à la fois un TOUT et une PARTIE d'un tout (effet Janus). Cette approche marque une rupture avec les modèles hiérarchiques antérieurs, où les comportements sont du type 'maître - esclave' selon une topologie arborescente et invariante des centres de décision, le tout renforcé par le respect des ordres par le centre de décision esclave. En effet, le holon a une intelligence décisionnelle qui lui permet d'agir sur son propre comportement, mais qui lui permet également d'intervenir sur le comportement du système auquel il appartient (Pujo and Ounnar, 2007). La décomposition hiérarchique est remplacée par la récursivité des holons et la mise en œuvre de l'effet Janus. Ceci permet une large latitude pour la mise en œuvre du système de pilotage, selon une architecture hétérarchique au sens large (Trentesaux, 2002), c'est-à-dire pouvant mixer des parties centralisées et des parties non centralisées.

Différentes architectures holoniques sont proposées dans la littérature scientifique pour le pilotage des HMS (Deen, 2003). La plus connue est PROSA (Product, Resource, Order, Staff Architecture) (Van Brussel et al, 1998). D'autres architectures sont extrêmement proches, comme Adacor (Leitao and Restivo, 2006), qui renforce substantiellement l'aspect hiérarchique, ou encore Metamorph (Balasubramanian et al., 2001), qui a rencontré un certain intérêt industriel et débouché sur la norme IEC 61499. Nous ne présenterons ici que PROSA.

\subsubsection{Concepts fondamentaux de PROSA}

PROSA comporte trois holons de base (figure 1).

Le Holon Produit (HP) contient le processus de fabrication du produit, c'est-à-dire la connaissance nécessaire pour assurer sa fabrication en obtenant une qualité suffisante. Il agit en tant que serveur de l'information pour les autres Holons du HMS, mais ne contient pas l'état du produit.

Le Holon Ressource (HR) contient une partie physique, à savoir l'équipement de production, et une partie de traitement de l'information qui commande l'équipement et contient des méthodes d'allocation des ressources.

Le Holon Ordre (HO) représente une tâche dans le système de production. Il est responsable de l'exécution du travail assigné dans les temps impartis. Il contrôle le produit physique, le modèle d'état de produit, et gère le traitement de l'information logistique lié au travail.

La gestion de la connaissance relative à la production résulte des interactions entre ces 3 types de holons. Les interactions HPHR donnent la connaissance du processus : méthodes d'exécution des ressources, capacité, qualité atteignable et résultats possibles... Les interactions HP-HO indiquent la connaissance de la production : descriptif des lots (quantités à livrer, référence du produit, échéancier de livraison...). Les interactions HR-HO apportent la connaissance de processus d'exécution : suivi de l'exécution des processus sur les ressources 'surveillance de la progression, de l'interruption du processus...).



Figure 1. Holons de base et connaissance associée (Van Brussel et al., 1998).

PROSA prévoit également la possibilité d'utiliser des Holons Staff pour aider les Holons de base dans l'exécution de leurs tâches, pour résoudre des situations de blocage ou rechercher une optimisation. Les Holons Staff n'ont pas de pouvoir de décision, mais peuvent proposer une solution globale pour résoudre un problème en fonction des données qui leur sont transmises par les Holons de base, qui restent responsables de la prise de décision finale. Toutefois, l'usage des Holons Staff reste très proche des 
comportements hiérarchiques, dans la mesure où la proposition de solution est élaborée de manière centralisée par le Holon Staff, selon des techniques somme toute très conventionnelles. Ensuite, cette solution sert de base comme programme prévisionnel que vont essayer d'exécuter les holons de base, comme dans une solution distribuée classique.

\subsection{Architecture isoarchique}

Nous travaillons depuis quelques années sur le problème de la conception de solutions de pilotage faisant abstraction des notions de hiérarchie et de centralisation (Pujo and Brun-Picard, 2002) (Pujo and Ounnar, 2007). L'étude de la décentralisation et de l'auto organisation nous a conduits à développer le concept d'isoarchie et à revisiter l'usage des concepts proposés par PROSA.

\subsubsection{Définition d'une architecture isoarchique}

Le terme 'isoarchie' est formé à partir de deux radicaux grecs : iso- (égal) et -archie (pouvoir), signifiant ainsi une même autorité et donc une totale absence de hiérarchie. Dans un système de décision composé de plusieurs centres de décision, une architecture décisionnelle peut être qualifiée d'isoarchique lorsque chaque centre de décision est doté de la même capacité de décision. Cette propriété peut être aisément obtenue lorsque les mécanismes de décision sont dupliqués sur chaque centre de décision et paramétrés à façon sur chacun. L'isoarchie apparaît alors comme une spécification particulière du concept d'hétérarchie et comme l'opposé absolu du concept de hiérarchie (Mesarović, et al., 1980). Au sens de (Trentesaux, 2002), elle peut être classée en décentralisée de type III (Déc, III) et ne doit souffrir d'aucune nuance. En effet, les discussions scientifiques autour des structures architecturales de pilotage se situent dans un espace linguistique allant du 'centralisé/hiérarchisé' au 'décentralisé/auto-organisé'. Cet espace inclut bien entendu toutes les solutions intermédiaires, décrites sous la base linguistique de 'semi-xx', 'pseudo-yy' ou encore 'zz partielle', 'xy au sens large', 'xz-supervisé'... Ceci a des dommages collatéraux, car il n'existe plus de terme exclusif à l'opposé de 'centralisation'. Par exemple, le terme 'hétérarchique', un temps candidat possible pour ce rôle, s'applique maintenant à des architectures distribuées sur plusieurs niveaux de décision, avec des décisions hiérarchiques locales. Il nous semble donc que le concept d'isoarchie ne doit être employé que sur des architectures réellement et totalement 'égalitaires'.

Ce concept d'isoarchie peut être mis en œuvre via le paradigme holonique.

\subsubsection{Mise en æuvre d'une approche holonique dans un contexte isoarchique}

Dans un système complexe composé d'entités ayant des capacités décisionnelles, l'isoarchie est caractérisée lorsque toutes les décisions sont prises grâce à l'autonomie de ces entités. Cela nécessite une capacité de communication directe entre ces entités, afin de pouvoir résoudre efficacement les problèmes de synchronisation, de coordination et/ou de coopération. La décentralisation des centres de décision offre cette possibilité : les entités décentralisées 'assurent en commun les décisions les concernant, sans consigne ou ordre émanant d'entités de niveau supérieur et grâce à des primitives fonctionnelles dupliquées sur chacune d'entre elles et en interactions via un protocole commun de communication' (Pujo and Brun-Picard, 2002). Ce schéma se retrouve également dans la notion de 'Flat Holonic Form' (Bongaerts et al. 2000), particularisation isoarchique d'une architecture holonique.

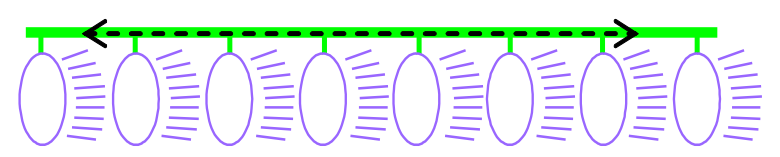

Figure 2. Flat Holonic Form

Par ailleurs, l'absence d'un système de décision central interdit toute organisation prédéfinie ou prévisionnelle du fonctionnement du système, qui va être organisé au fur et à mesure par les entités. Cette auto-organisation sous-entend un caractère temps réel qui prend en considération toutes les informations caractérisant chacune des entités contribuant à l'établissement de ce fonctionnement. Nous parlons alors de fonctions de pilotage auto-organisé. Ces fonctions sont intégrées dans l'intelligence associée à chacune des entités que nous appelons holons. Nous définissons un Holon comme une entité conceptuelle reposant sur l'association d'une Structure Matérielle (le M_holon), d'un Système d'Information et d'un système de traitement (le I_holon), le dotant d'une Intelligence Décisionnelle lui permettant de fonctionner en interaction avec d'autres holons (figure 3). Cette structuration permet une décomposition récursive des systèmes de production, en accord avec le paradigme holonique, en faisant clairement apparaitre la dualité et le parallélisme entre monde réel (matériel) et informationnel (immatériel, où se situent données et prises de décision). Nous retrouvons les notions de Holon Produit, Ressource et Ordre. 


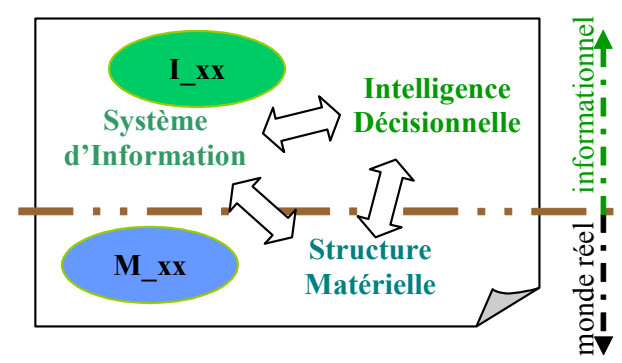

Figure 3. Structure basique d'un holon $X X$

Un Holon Produit (HP) est constitué d'un M_produit (l'objet physique) et d'un I_produit qui contient le processus de fabrication du produit, mais aussi son modèle d'état et toutes les informations concernant sa traçabilité, en d'autres termes et respectivement son futur, son présent et son passé (Pannequin and Thomas, 2006). Il existe donc autant d'instances de HP que de produits fabriqués ou en cours de fabrication. Ceci est une différence majeure avec PROSA. Cette identification à l'unité passe par le déploiement des technologies infotroniques ad hoc reliant chaque M_produit à son I_produit. Un bon exemple de ces technologies est le RFID, avec un tag ID attaché au M_produit et contenant les informations clefs du I_produit.

Un Holon Ordre $(\mathrm{HO})$ représente une tâche dans le système de production : un ordre de fabrication concernant généralement un ensemble de HP. Il est donc intimement lié aux notions de lot, d'encours et de délais. Le I_ordre veille lors de l'exécution du travail au respect des dates ainsi qu'à la prise en compte de facteurs économiques (taille du lot, volume de l'encours, minimisation des changements de production, fractionnement d'un lot...). Le M_ordre sera selon les cas l'OF papier, muni d'un tag ID, ou le conteneur, également tagué, qui permet la manipulation des M_produits.

Le Holon Ressource (HR) reste conceptuellement similaire à la définition de PROSA. La M_ressource correspond à un équipement automatisé standard actuel : MOCN, robot industriel... Bien entendu, les méthodes d'allocation des ressources de la I_ressource évoluent, puisque les interactions avec les autres types de holons sont différentes dans notre contexte isoarchique.

Une autre différence majeure avec PROSA est la disparition du Holon Staff, qui n'a plus de raison d'être dans un contexte isoarchique. Nous lui substituons un Holon Simulation ayant un objectif totalement différent : il s'agit, à partir de l'état du système de production, obtenu par l'écoute et l'analyse des interactions entre tous les autres holons, de simuler l'évolution du système de production et d'anticiper d'éventuels dysfonctionnements. Ce holon ne contribue pas à l'auto organisation mais facilite le rôle du pilote de l'atelier : il confère au pilotage des propriétés proactives (Pujo et al., 2004) que nous ne développerons pas ici.

In fine, notre approche est appelée PROSIS, pour Product, Resource, Order, \& Simulation Isoarchic System.

\subsubsection{Entité de Pilotage Autonome}

L'auto-organisation d'un tel ensemble de holons sous entend que les décisions de pilotage doivent être prises localement. PROSIS reflète naturellement l'organisation physique des entités : autour de chaque ressource se retrouvent physiquement une M_ressource, $p$ M_produits et $k$ M_ordres. Ces derniers concernent les p produits correspondants $(k \leq p)$. La prise de décision auto-organisée dans le pilotage exige la participation de toutes les entités impliquées : ressource, ordres et produits. Pour cela, des interactions locales et spécifiques seront établies entre les I_holons des $(1+p+k)$ holons concernés : une I_ressource, $p$ I_produits et $k$ I_ordres. Ces I_holons constituent une 'Flat Holonic Form' locale. Il apparait clairement que ces holons n'ont pas tous le même objectif : un compromis doit être trouvé, faisant émerger une bonne solution de pilotage. Pour gérer cela, une Entité de Pilotage Autonome (EPA), fournissant aux I_holons différents services ad hoc, est associée à chaque ressource. Une EPA permet l'accueil des $(1+p+k)$ I_holons, favorise leurs interactions et actualise l'état des informations relatives au WIP de chaque ressource. Pour cela, chaque EPA est dotée d'un coupleur RFID, qui permet la synchronisation entre le monde physique et le système d'information : quand un produit ou un lot de produits arrive dans l'encours de la ressource (ou le quitte), le ID tag correspondant est lu et l'ensemble \{I_produit, I_ordre\} est actualisé (ajouté ou retiré de la structure d'information de l'EPA). Une EPA est également dotée d'un ensemble de fonctions visant à organiser les interactions entre les I_holons, de manière à favoriser la productivité selon des critères qui peuvent être paramétrés. Le problème de l'auto-organisation repose sur la manière de piloter en temps réel les flux de produits. Dans le cas où les flux sont définis par les gammes des produits, les EPA reliées en réseau s'échangent des informations relatives à ces flux. Le vrai problème consiste à la régulation temporelle de ces flux. Ceci est l'un des services fondamentaux supporté par l'EPA : proposer aux I_holons, sur la base des informations les concernant, le choix du prochain produit à traiter, choisi dans le WIP et contribuant à l'obtention d'une solution 'contextuellement' optimale. PROSIS permettrait de traiter ce problème avec des heuristiques tout à fait conventionnelles, principalement monocritère. Pour exploiter pleinement le potentiel de PROSIS, nous nous sommes orientés vers l'utilisation de méthodes d'aide à la décision multicritère. Les données nécessaires à la mise en œuvre de la méthode choisie sont issues des interactions précédemment évoquées entre les holons concernés. 


\section{Décision multicritère en pilotage holonique et isoarchique}

L'approche par analyse multicritère permet de prendre en compte et/ou de concilier des intérêts parfois contradictoires. Après un rapide panorama de ces méthodes justifiant notre choix, nous montrons comment s'élaborent localement les prises de décision de pilotage.

\subsection{Méthodes d'analyse et de décision multicritères}

\subsubsection{Panorama des méthodes d'analyse et de décision multicritères}

Nous pouvons distinguer trois classes de méthodes multicritères : les méthodes d'aide à la décision multicritère, les méthodes élémentaires et les méthodes d'optimisation mathématiques. Le choix de l'une ou de l'autre des trois classes de méthodes peut dépendre soit des données dont nous disposons pour traiter le problème multicritère considéré, soit de la façon avec laquelle le décideur modélise ses préférences. Dans notre cas, le processus de choix du produit devant être traité par une ressource suppose une connaissance des différentes alternatives possibles pour effectuer un tri par rapport à un ensemble de critères. De ce fait, l'utilisation des méthodes d'optimisation mathématiques n'est pas possible. D'autre part, l'objectif étant d'effectuer un classement, les méthodes élémentaires ne sont pas considérées. Les méthodes d'aide à la décision multicritère permettent d'apporter une aide au décideur au cours de l'affinement de son processus de décision qui porte sur le choix d'une alternative parmi un ensemble d'alternatives potentielles. L'ensemble des produits dans la file d'attente (encours) de la ressource constitue l'ensemble des alternatives. Il s'agit d'effectuer le classement de l'ensemble des alternatives, en examinant la logique, la cohérence des choix, puis en agrégeant les préférences, selon l'une des trois approches complète, partielle ou locale. Dans notre cas, l'agrégation complète est appliquée pour classer l'ensemble des produits de l'encours d'une ressource. Plusieurs méthodes existent (Ounnar, 1999) (Mekaouche, 2007) : TOPSIS (Technique for Order Preference by Similarity to Ideal Solution), MAVT (Multiple Attribut Value Theory), MAUT (Multiple Attribut Utility Theory), SMART (Simple Multiple-Attribut Rating Technique), UTA (Utility Theory Additive), EVAMIX (EVAluation of MIXed criteria), AHP (Analytic Hierarchy Process). Nous avons choisi AHP pour ses propriétés structurantes et formalisantes.

\subsubsection{AHP (Analytic Hierarchical Process)}

La méthode AHP (Saaty, 1980) décompose un problème complexe de décision en un ou plusieurs niveaux de détails où l'évaluation des valeurs est fournie par des comparaisons par paires. Contrairement aux autres méthodes, AHP est la seule qui permet d'une part la mesure de la cohérence des préférences du décideur et d'autre part la prise en compte à la fois de l'indépendance et de l'interdépendance des critères d'évaluations considérés. La prise en compte de l'interdépendance se traduit par la construction d'une structure hiérarchique reflétant ainsi les différents niveaux selon les relations liants les critères. Les éléments de la hiérarchie (critères, sous critères, alternatives) ne sont pas forcément liés entre eux, néanmoins ils peuvent être groupés dans des ensembles disjoints. Par conséquent, cette méthode permet l'utilisation de listes complètes de critères d'évaluation sans en exclure aucun. Notons également que la méthode AHP permet la prise en compte de critères qualitatifs et quantitatifs. Toutes ces caractéristiques constituent les points forts de la méthode AHP (Ounnar et al., 2007). Dans le but de fournir une approche structurante, des vecteurs de priorités sont établis en utilisant des mesures de cohérence. La méthode AHP est donc un processus de mesure à l'intérieur de structures ou de réseaux hiérarchiques, permettant d'exprimer ses préférences de choix parmi plusieurs critères et alternatives en ce qui concerne chaque critère et ensuite, d'obtenir un classement d'ensemble des alternatives utilisant les poids des critères. AHP est donc un processus de prise de décision qui interprète directement des données et des informations et surtout qui peut être totalement automatisé.

Un algorithme de décision multicritère appliquant AHP est donc implanté dans chaque EPA : c'est un outil mis à la disposition des holons par l'EPA, utilisé en fonction des besoins de ces holons et permettant de définir un classement des HP prenant en compte les différentes contraintes des HR, HP et HO. Pour un HR venant d'achever le traitement d'un M_produit, seul le I_produit classé en première position est intéressant, car il correspond au prochain $\mathrm{M}$ _produit à traiter par la ressource. Pour un HP quelconque, l'algorithme peut lui donner une indication sur sa probabilité de traitement plus ou moins rapide, et le HP peut éventuellement modifier certains de ses paramètres. L'algorithme AHP se met en œuvre en deux grandes phases que nous allons maintenant détailler.

\subsubsection{Configuration de l'algorithme AHP}

Pour pouvoir utiliser l'algorithme AHP pour classer les produits situés dans les files d'attente, il faut au préalable régler le paramétrage de l'importance relative des critères et de leurs indicateurs. Ceci configure la comparaison par paires entre les différents critères $C_{j}$ par rapport à leur importance dans la décision de choix du HP. Cette phase 'statique' de l'algorithme (c'est-àdire que cela sera effectué une seule fois pour toute pour chaque algorithme implanté sur une EPA, en fonction du HR et de l'ensemble des HPs pouvant le traverser) doit être validée par une vérification mathématique de cohérence.

Tout d'abord, il s'agit de classer les critères par rapport à un objectif global. Nous construisons pour cela une matrice [C] où chaque élément $c_{i j}$ est un jugement ou une comparaison entre une paire de critères $C_{i}$ et $C_{j}$, selon une échelle $1-9$, avec $c_{i j}=1 / c_{j i} \&$ $\mathrm{c}_{\mathrm{ii}}=1$. Cette matrice permet de déterminer le vecteur d'importance relative $\left[\mathrm{V}_{\mathrm{COg}}\right]$. Des vérifications de cohérence permettent de détecter et de corriger les poids affectés : toute incohérence constatée nécessitant une redéfinition des paramètres d'importance relative. 
Ensuite, il s'agit, vis-à-vis de l'ensemble des critères $\mathrm{C}_{\mathrm{k}}$, d'établir un classement de chacun des indicateurs $\mathrm{I}_{\mathrm{k}, \mathrm{i}}$ par rapport à son critère $C_{k}$. A cet effet, nous construisons une matrice $\left[I_{C k}\right]$ où chaque élément $i_{k, i j}$ est un jugement ou une comparaison entre une paire d'indicateurs $I_{k, i}$ et $I_{k, j}$. Pour chaque matrice $\left[I_{C k}\right]$, nous estimons le vecteur d'importance relative [ $V_{\text {ICk }}$ selon le même principe, avec validation de la cohérence de la matrice $\left[\mathrm{I}_{\mathrm{Ck}}\right]$.

\subsubsection{Exploitation de l'algorithme AHP}

La phase d'exploitation dynamique de l'algorithme AHP permet de classer les HPs de la file d'attente. Premièrement, il s'agit d'établir un classement des HPs par rapport à chaque indicateur $\mathrm{I}_{\mathrm{kx}}$ de chaque critère $\mathrm{C}_{\mathrm{k}}$. Pour chaque indicateur, nous comparons deux à deux les valeurs de l'indicateur entre les HPs de la file d'attente. Nous pouvons ainsi construire une matrice [ $\left.\mathrm{P}_{\mathrm{kx}}\right]$, avec : $\mathrm{p}_{\mathrm{kx} ; \mathrm{ij}}=\mathrm{I}_{\mathrm{kx}, \mathrm{j}} / \mathrm{I}_{\mathrm{kx}, \mathrm{i}}$ pour minimiser le critère et $\mathrm{p}_{\mathrm{kx} ; \mathrm{ij}}=\mathrm{I}_{\mathrm{kx}, \mathrm{i}} / \mathrm{I}_{\mathrm{kx}, \mathrm{j}}$ pour le maximiser, $\mathrm{p}_{\mathrm{kx} ; \mathrm{ij}}=1 / \mathrm{p}_{\mathrm{kx} ; \mathrm{ji}}$ et $\mathrm{p}_{\mathrm{kx} ; \mathrm{i}}=1$. Nous appellerons $\left[\mathrm{V}_{\mathrm{PIk}, \mathrm{x}}\right]$ le vecteur d'importance relative entre PHs qu'il est alors possible de calculer.

Ensuite, il faut remonter dans la hiérarchie de choix relatifs, car le but est de déterminer l'importance relative des HPs par rapport à l'objectif global. La matrice $\left[\mathrm{PI}_{\mathrm{k}}\right]$ est construite, où chaque colonne est un vecteur $\left[\mathrm{V}_{\mathrm{PIk}, \mathrm{x}}\right]$. Un vecteur donnant l'importance relative des produits par rapport aux critères est construit, et ceci, pour chacun des critères : $\left[\mathrm{PC}_{\mathrm{k}}\right]=\left[\mathrm{PI}_{\mathrm{k}}\right] *\left[\mathrm{~V}_{\mathrm{ICk}}\right]$; Les vecteurs $\left[\mathrm{PC}_{\mathrm{k}}\right]$ permettent de construire une matrice $[\mathrm{PC}]=\left[\mathrm{PC}_{1}, \mathrm{PC}_{2}, \ldots, \mathrm{PC}_{\mathrm{n}}\right]$. Le produit $[\mathrm{PC}] *\left[\mathrm{~V}_{\mathrm{Cog}}\right]$ fournit le vecteur de priorité $\left[\mathrm{V}_{\mathrm{POg}}\right]$ des HPs considérés, dont la plus grande composante $\mathrm{VPOg}_{\mathrm{g}}$ correspond au $\mathrm{HP}_{\mathrm{y}}$ à choisir.

\subsection{Analyse des différentes sources d'intérêt}

Pour construire le système hiérarchique de critères et identifier les indicateurs correspondants, nous allons maintenant exposer les différents points de vue à analyser conjointement pour respecter les différents intérêts des holons en interaction dans PROSIS.

\subsubsection{Intérêts du point de vue 'Holon Ressource'}

Une ressource doit rechercher avant tout la productivité, qui minimise les coûts d'exploitation de la ressource. Ceci peut être évalué au travers d'un indicateur tel que le REP (Ratio d'Efficacité du Processus), qui doit être maximum. Une stratégie consiste à éviter de laisser la ressource à l'arrêt, et pour cela à compléter la charge correspondant à la production principale par une production secondaire, forcément moins prioritaire.

Un premier critère $\mathrm{C} 1$, liant chaque HR aux HP, permet de prioriser certains types de production, en fonction de leur appartenance à différentes classes : ressource principale (ressource dédiée au produit) ou secondaire (ressource de remplacement en cas d'indisponibilité d'une ressource équivalente), produit faisant ou non l'objet d'un volume de production substantiel et géré selon une approche 'ligne de production', produit pour un assemblage d'appareils neufs ou produit pour pièce de rechange... Chacun des indicateurs associés (I11, I12, I13) sont des indicateurs qualitatifs, définis par des valeurs actualisées périodiquement par le management de l'atelier.

Une autre idée intéressante est d'éviter de travailler sans efficacité. Plus la charge d'une ressource est élevée, plus il devient judicieux de privilégier les produits de priorité élevée. De même, il n'est pas judicieux de traiter un produit de priorité faible ou moyenne sur une ressource si la ressource suivante le concernant est surchargée. Le critère C5 est relatif au taux d'occupation de la ressource et permet de prendre ces contraintes en considération. Dans les autres cas, ce critère n'influencera pas le classement obtenu par l'algorithme AHP. L'indicateur I51, associé à ce critère, quantifie le cumul des tâches en attente dans l'encours de la machine.

\subsubsection{Intérêts du point de vue 'Holon Produit'}

Le coût minimal d'obtention est un objectif fondamental à atteindre pour un HP. Comme les temps technologiques de traitement sont généralement optimisés par les réglages ad hoc du procédé, nous ne pouvons agir efficacement qu'en minimisant la durée d'attente du produit. Si cela est réalisé systématiquement dans tous les encours traversés par les produits, nous obtenons le cycle moyen d'obtention le plus court possible, et donc les coûts d'immobilisation les plus faibles.

Pour répondre à cet objectif, nous avons développé une stratégie visant à créer artificiellement un effet de flux tiré. Le critère C2 permet de favoriser les HP dont l'état d'avancement est proche de l'état final. Soit n, le nombre de phases de traitement à effectuer sur un HP et $\mathrm{k}$ la phase actuelle du HP. Plus le HP est proche de son achèvement $(\mathrm{k} / \mathrm{n}$ proche de 1$)$, plus la priorité de ce HP devra être importante. L'indicateur I21 $(\mathrm{I} 21=\mathrm{k} / \mathrm{n})$, correspondant à la progression globale du produit, permet de dynamiser l'écoulement des flux tout au long de la 'ligne virtuelle de production' associée à sa gamme. Cet appel par l'aval est suscité par le HP lui-même. Autrement dit, les HP contribuent prioritairement à vider le système de production, ce qui libère de la capacité que les HR se chargent d'utiliser. De même, toujours pour minimiser les délais d'attente, un indicateur de progression locale I22 favorise l'écoulement des flux d'un HP s'il s'avère que le HR suivant concernant cet HP le traitera sûrement dans la foulée. Ceci n'implique aucun engagement prévisionnel. Enfin, différents produits de priorités diverses circulent simultanément dans le système de production. Un risque non négligeable serait 'd'enterrer' un produit de faible priorité dans une file d'attente et de ne plus pouvoir l'en sortir. Le critère C4 et son indicateur I41, constitué de la durée d'attente du HP devant un HR, vise à le sortir de la file d'attente s'il y séjourne depuis trop longtemps, en augmentant pour cela, son importance relative. 


\subsubsection{Intérêts du point de vue 'Holon Ordre'}

Du point de vue du holon ordre, l'objectif fondamental est le respect des dates de livraison. C'est un aspect assez classique en ordonnancement. Notre critère C3 examine la marge restante pour chaque HP : dans le cas où le nombre d'opérations restantes sur un HP est important et que la date de livraison de l'HO auquel il est rattaché approche, il devient urgent de le prioriser vis-à-vis d'autres HP. Ce critère permet donc de respecter les dates de livraison d'un HO. Pour un HO constitué de plusieurs HP, nous pouvons considérer qu'une grande partie des HP sera traitée dans les temps, mais qu'il pourra subsister quelques HP 'retardataires' : ce critère vise à accélérer le traitement de ces derniers. L'indicateur associé I31 est donné par la formule : I31 = durée_restante - durée_des_phases_restantes. Plus I31 est petit, plus le HP concerné sera priorisé.

Bien entendu, d'autres indicateurs pourraient être utilisés comme par exemple une marge restante pondérée (I31/nombre_de_phases_restantes), qui augmenterait la prise en compte de ce critère.

\subsubsection{Structure hiérarchique de décision choisie}

L'ensemble des choix de critères et d'indicateurs associés énoncés ci-dessus permet de mettre en place la structure hiérarchique de décision illustrée en figure 4.

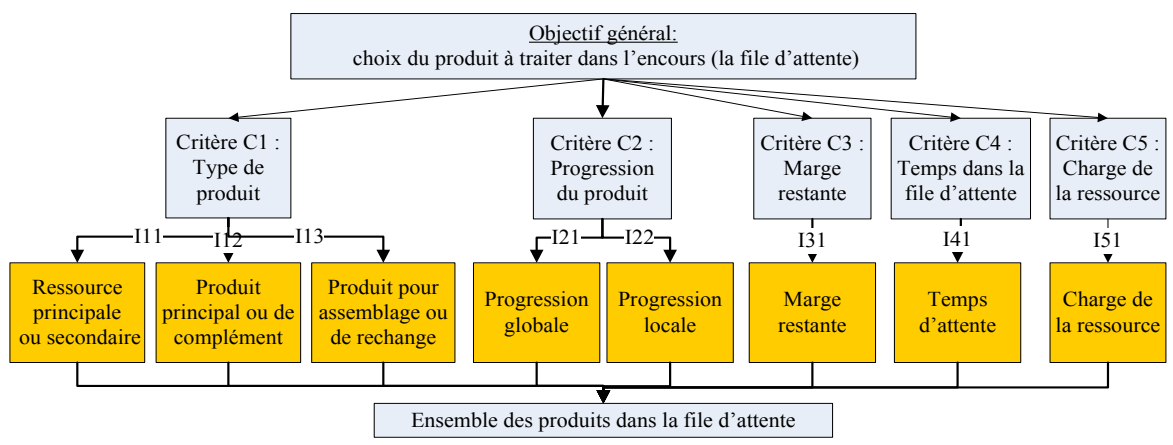

Figure 4. Structure hiérarchique de décision multicritères

\section{Cas d'étude}

Le cas d'étude présenté ici est issu de l'industrie aéronautique. Après une présentation sommaire de l'unité de production ayant fourni le jeu d'essai, nous présentons le modèle construit sous Arena, visant à montrer la faisabilité de l'approche et les résultats obtenus sous différentes conditions d'expérimentation.

\subsection{Présentation de l'Unité 4-5}

Le système de production test, l'Unité 4-5, est un atelier de production mécanique fabricant de manière récurrente une vingtaine de types différents de pièces, principalement des 'arbres' et des 'manchons' de grandes tailles, ayant des tolérances serrées et des gammes complexes et étant au cœur des systèmes aéroportés fabriqués par l'entreprise. Cet atelier comporte une quinzaine de machines de type centre de production CNC polyvalent de haute précision, de grandes capacités et dotés de tous les équipements périphériques ad hoc. La taille des lots dépasse rarement une quinzaine de pièces, chaque pièce représentant une valeur de plusieurs centaines de milliers d'euros. Le cycle d'encours peut atteindre pour les productions les plus complexes 6 mois.

Pour des raisons de confidentialité, il nous est impossible de décrire plus finement le cas d'essai.

Différents problèmes sont observés dans l'Unité 4-5. Le pilotage de l'atelier est issu d'un calcul MRP classique, associé à des tailles de lot obtenues via des calculs d'optimisation économiques qui n'ont plus lieu d'être, vu l'utilisation récentes de machines palettisées où les tâches de préparation sont effectuées en temps masqué. Nous constatons des temps de cycle trop longs, certaines pièces pouvant rester jusqu'à 6 mois dans l'atelier. Ceci engendre des retards de livraison chroniques. Par ailleurs, un manque de synchronisation laisse les ressources sans occupation une partie du temps, et le REP est faible ( $<$ à $50 \%)$.

\subsection{Maquette de test}

\subsubsection{Modélisation de l'Unité 4-5}

Pour développer un modèle de simulation de l'atelier, nous avons choisi d'utiliser un outil de simulation industrielle, Arena 8.01. Ce modèle reprend l'organisation générale des flux entre les ressources. La figure 5 montre la partie 'production type arbre' 
de 1'Unité 4-5.

Le modèle de chaque HR permet, à partir d'une variable booléenne définie en début de simulation, soit d'utiliser une discipline FIFO dans la file d'attente, soit d'utiliser la discipline issue de l'algorithme AHP. Cette possibilité de choix d'expérimentation est illustrée par la figure 6 .

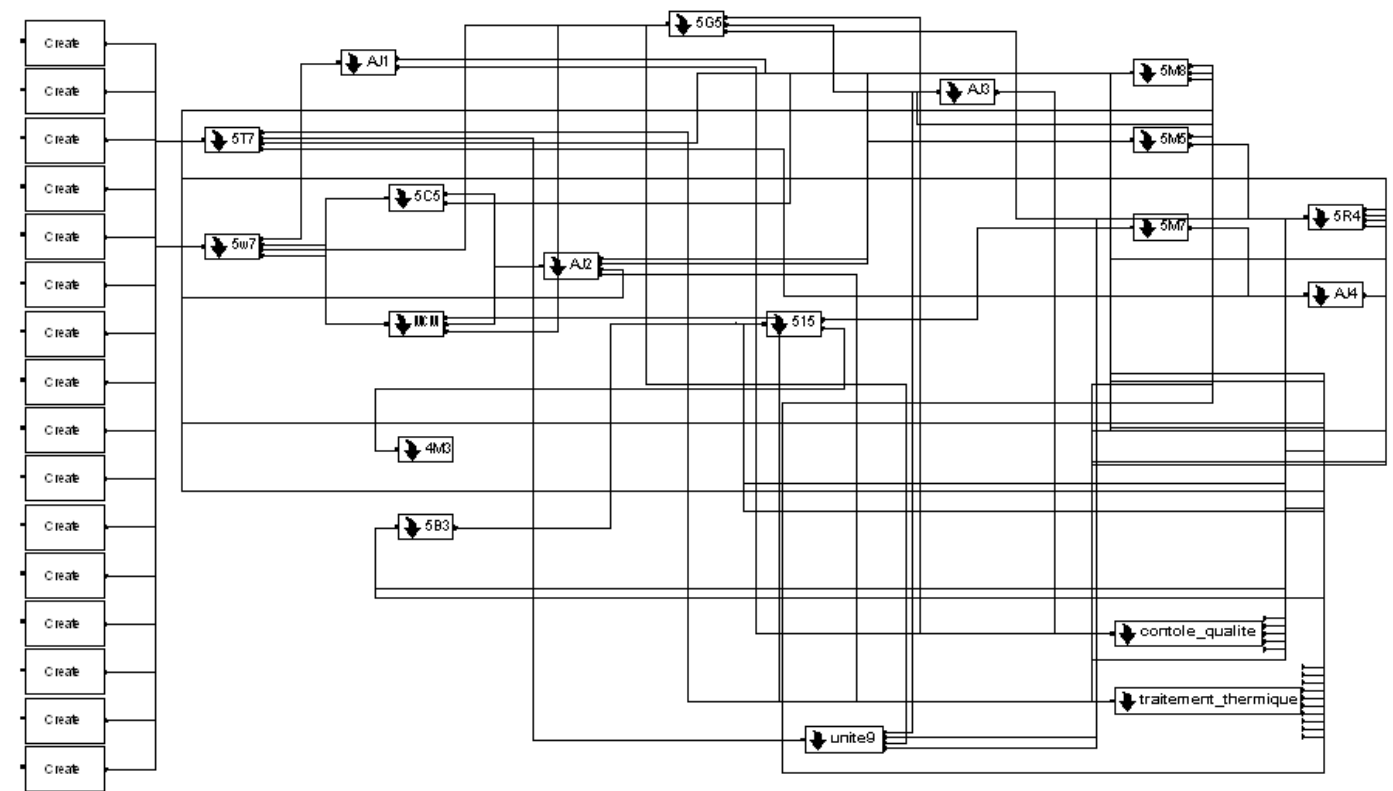

Figure 5. Modèle de simulation sous Arena 8.01

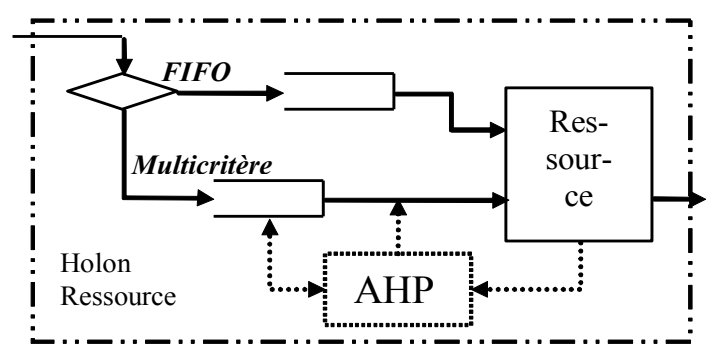

Figure 6. Choix d'expérimentation

\subsubsection{Mise en ceuvre du modèle}

Nous allons maintenant régler le système hiérarchique de critères et d'indicateurs correspondant, puis montrer comment l'alimenter en données à partir d'Arena. Il faut commencer par la configuration de l'algorithme AHP. Chaque ressource a son propre jeu de pondération. La matrice d'importance relative des critères [C] (exemple donné au tableau 1) est constante sur tous les RH. Il en est de même pour les matrices d'importance relative $\left[\mathrm{I}_{\mathrm{Ck}}\right]$ entre les indicateurs $\mathrm{I}_{\mathrm{C} 1}$ et $\mathrm{I}_{\mathrm{C} 2}$. Par contre, les matrices $\left[\mathrm{P}_{\mathrm{kx}}\right]$ d'importance relative entre alternatives, pour un indicateur ont leur dimension qui varie en fonction du nombre de HP dans l'encours. Cette dimension dépend déjà du nombre de types de produits différents habilités à passer par la ressource.

\begin{tabular}{c|c|c|c|c|c} 
& $\mathrm{C} 1$ & $\mathrm{C} 2$ & $\mathrm{C} 3$ & $\mathrm{C} 4$ & $\mathrm{C} 5$ \\
\hline $\mathrm{C} 1$ & 1 & $1 / 3$ & $1 / 3$ & 3 & 1 \\
\hline $\mathrm{C} 2$ & 3 & 1 & 1 & 9 & 3 \\
\hline $\mathrm{C} 3$ & 3 & 1 & 1 & 9 & 3 \\
\hline $\mathrm{C} 4$ & $1 / 3$ & $1 / 9$ & $1 / 9$ & 1 & $1 / 3$ \\
\hline $\mathrm{C} 5$ & 1 & $1 / 3$ & $1 / 3$ & 3 & 1 \\
\hline
\end{tabular}

Tableau 1. Matrice d'importance relative entre critères [C]

Les éléments $p_{\mathrm{kx} ; \mathrm{ij}}$ sont directement calculés à partir des valeurs des indicateurs, qu'ils soient eux-mêmes des valeurs prédéfinies ou des valeurs calculées à partir des attributs de certains composants du modèle de simulation. 
Le tableau 2 montre un exemple de matrice $\left[\mathrm{P}_{\mathrm{kx}}\right]$, après récupération des données Arena pour construire les indicateurs et calculs des ratios $p_{\mathrm{kx} ; \mathrm{ij}}$. Nous y observons que des produits pouvant être traités par la ressource peuvent être absents de l'encours sans aucun problème.

\begin{tabular}{|c|c|c|c|c|c|c|c|c|c|c|c|c|c|c|c|c|}
\hline & P1 & P2 & P3 & P7 & P11 & P12 & P21 & P27 & P28 & P32 & P34 & P35 & P37 & P43 & P45 & P49 \\
\hline P1 & 1 & 0,1 & 0,12 & 0,5 & 0,14 & 1 & 1 & 1 & 1 & 1 & 1 & 1 & 1 & 1 & 1 & 1 \\
\hline P2 & 10 & 1 & 1,25 & 5 & 1,43 & 10 & 10 & 10 & 10 & 10 & 10 & 10 & 10 & 10 & 10 & 10 \\
\hline P3 & 8 & 0,8 & 1 & 4 & 1,14 & 8 & 8 & 8 & 8 & 8 & 8 & 8 & 8 & 8 & 8 & 8 \\
\hline P7 & 2 & 0,2 & 0,25 & 1 & 0,28 & 2 & 2 & 2 & 2 & 2 & 2 & 2 & 2 & 2 & 2 & 2 \\
\hline P11 & 7 & 0,7 & 0,87 & 3,5 & 1 & 7 & 7 & 7 & 7 & 7 & 7 & 7 & 7 & 7 & 7 & 7 \\
\hline P12 & 1 & 0,1 & 0,12 & 0,5 & 0,14 & 1 & 1 & 1 & 1 & 1 & 1 & 1 & 1 & 1 & 1 & 1 \\
\hline P21 & 1 & 0,1 & 0,12 & 0,5 & 0,14 & 1 & 1 & 1 & 1 & 1 & 1 & 1 & 1 & 1 & 1 & 1 \\
\hline P27 & 1 & 0,1 & 0,12 & 0,5 & 0,14 & 1 & 1 & 1 & 1 & 1 & 1 & 1 & 1 & 1 & 1 & 1 \\
\hline P28 & 1 & 0,1 & 0,12 & 0,5 & 0,14 & 1 & 1 & 1 & 1 & 1 & 1 & 1 & 1 & 1 & 1 & 1 \\
\hline P32 & 1 & 0,1 & 0,12 & 0,5 & 0,14 & 1 & 1 & 1 & 1 & 1 & 1 & 1 & 1 & 1 & 1 & 1 \\
\hline P34 & 1 & 0,1 & 0,12 & 0,5 & 0,14 & 1 & 1 & 1 & 1 & 1 & 1 & 1 & 1 & 1 & 1 & 1 \\
\hline P35 & 1 & 0,1 & 0,12 & 0,5 & 0,14 & 1 & 1 & 1 & 1 & 1 & 1 & 1 & 1 & 1 & 1 & 1 \\
\hline P37 & 1 & 0,1 & 0,12 & 0,5 & 0,14 & 1 & 1 & 1 & 1 & 1 & 1 & 1 & 1 & 1 & 1 & 1 \\
\hline P43 & 1 & 0,1 & 0,12 & 0,5 & 0,14 & 1 & 1 & 1 & 1 & 1 & 1 & 1 & 1 & 1 & 1 & 1 \\
\hline P45 & 1 & 0,1 & 0,12 & 0,5 & 0,14 & 1 & 1 & 1 & 1 & 1 & 1 & 1 & 1 & 1 & 1 & 1 \\
\hline P49 & 1 & 0,1 & 0,12 & 0,5 & 0,14 & 1 & 1 & 1 & 1 & 1 & 1 & 1 & 1 & 1 & 1 & 1 \\
\hline
\end{tabular}

Tableau 2. Exemple de matrice $\left[P_{k x}\right]$ entre 16 alternatives possibles (5 produits effectivement dans l'encours).

\subsubsection{Instrumentation des données}

La simulation sous Arena est couplée à un module de calcul développé via des feuilles de calcul Excel. Les différentes données nécessaires (HP, HO), concernant les produits en attente dans l'encours, sont recueillies dans le modèle Arena, puis exportées dans une feuille Excel propre à chaque HR. A chaque cellule correspondent des attributs attendus pour le calcul. L'algorithme AHP du HR est programmé dans le même fichier Excel. L'introduction de nouvelles données dans la feuille lance l'actualisation des calculs. Le résultat du classement multicritères est alors réimporté dans le modèle Arena, et sert à sélectionner le bon HP dans la file d'attente, pour continuer ensuite la simulation. Le produit correspondant est alors traité par le modèle de simulation de la ressource. Ce mécanisme d'aller retour entre Arena et Excel est illustré par la figure 7.

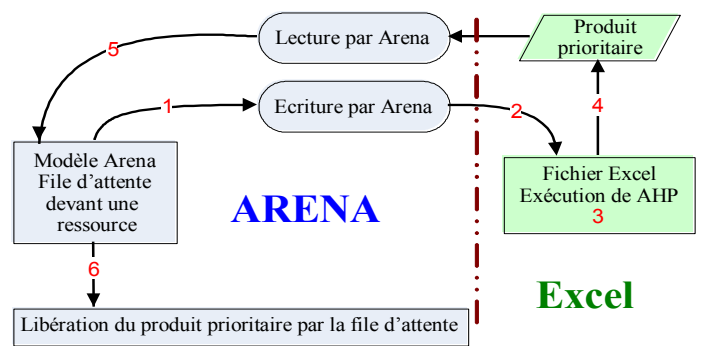

Figure 7. Mécanisme d'appel à l'algorithme AHP

Cette approche de modélisation intègre, au sens de (Pfeiffer et al., 2003), dans un même modèle le système opérant et le système de pilotage. Ceci est suffisant pour une première évaluation de l'approche.

\subsection{Expérimentation}

Les expérimentations conduites via le modèle présenté ci-dessus ont utilisées un jeu de données correspondant à 18 mois de production réelle de l'Unité 4-5. Cette période témoin est suffisamment grande pour témoigner de la diversité des situations de production. Par ailleurs, la période étant déjà achevée, la performance réelle de l'unité 4-5 en termes de productivité était connue. Les noms des produits et des ressources ont été rendus anonymes. Nous avons donc utilisé les mêmes dates de lancement que celles utilisées en production réelle. La seule modification vient de la transformation des lots $i$ de taille $n_{i}$ en $n_{i}$ lots de taille unitaire. Ceci revient à favoriser une approche de production pièce/pièce, plus fluide que la traditionnelle approche par lots économiques qui génère des problèmes de synchronisation de tâches qui dégradent le REP. Les expérimentations ont été conduites dans les conditions décrites au §4.2.1 avec comparaison systématique entre les deux approches. Dans le cas d'expérimentations conduites en discipline FIFO, les produits sont traités séquentiellement par la ressource, selon l'ordre d'arrivée dans le stock 
d'encours. Rien que le passage à une stratégie 'lots de taille 1' donne une meilleure productivité que la meilleure des heuristiques d'ordonnancement par lots utilisée dans l'Unité. Pour des raisons de confidentialité, nous ne pouvons donner ici de valeurs quantitatives. Dans le cas d'expérimentations en discipline multicritère, les choix du holon produit à traiter par chaque holon ressource se fait en temps réel, c'est-à-dire durant l'instant courant de simulation. Lorsque la ressource achève un produit se pose alors le choix du produit suivant, avec appel à l'algorithme AHP.

\subsection{Résultats obtenus}

\subsubsection{Comparaison entre les deux approches}

La figure 8 présente les résultats moyens sur la période de 18 mois : il s'agit de la moyenne des temps de passage des pièces dans l'Unité 4-5, par type de pièces. Ne sont indiquées que les produits de type 'arbre'. Les pièces de type 'manchon' donnent des résultats similaires et les pièces de complément ne sont pas concernées par des critères de productivité. Par ailleurs, il est à noter que les résultats en mode FIFO présentent des indicateurs de performances tout à fait comparables à ceux obtenus à partir du fonctionnement réel de l'Unité 4-5. Ces résultats montrent la réduction du temps de cycle de la quasi-totalité des produits. Seule une anomalie apparaît sur le produit 14. Après vérification, ce produit correspond à une production marginale, en faible quantité, pour le retrofit d'anciens appareils.

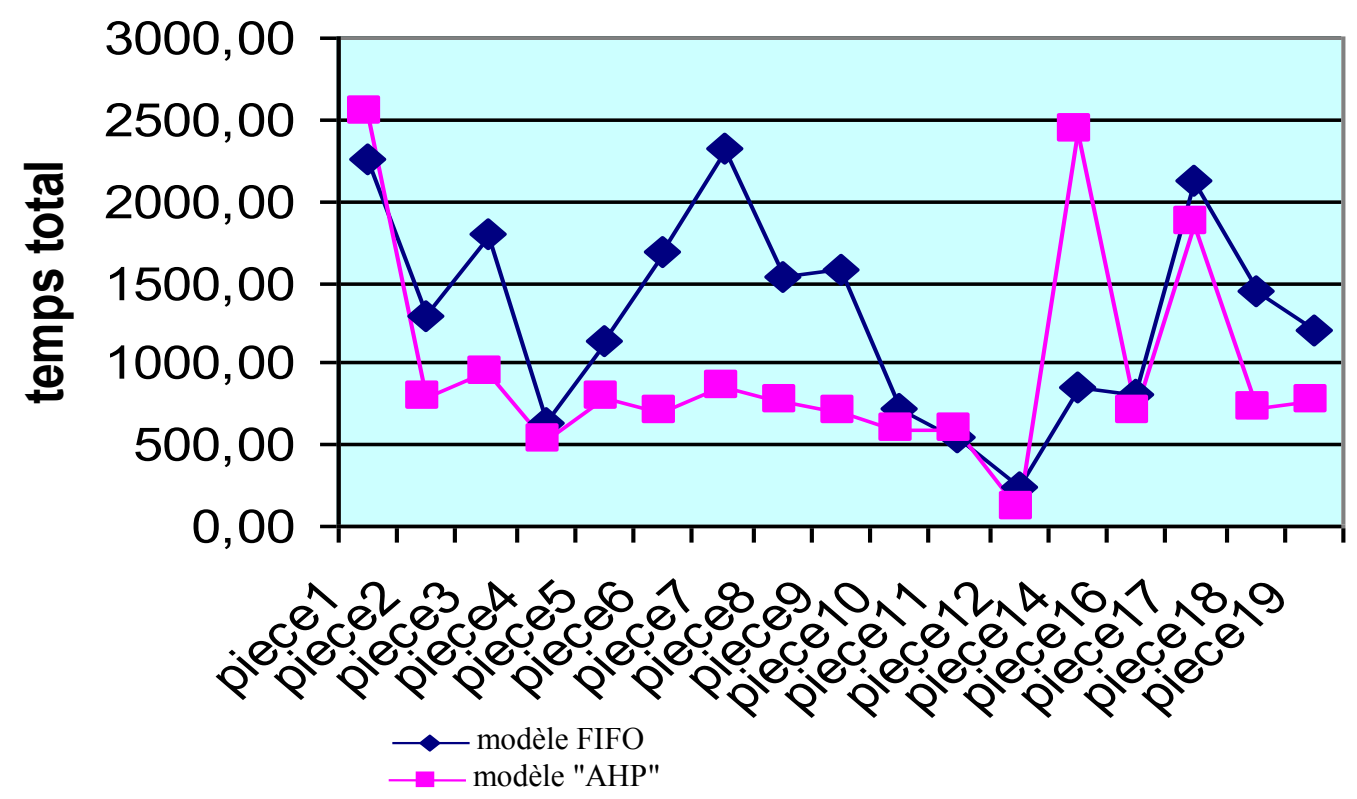

Figure 8. Comparaison des résultats selon les 2 disciplines de gestion des encours.

\subsubsection{Analyse des résultats}

Le tableau 3 présente la synthèse des résultats obtenus, c’est-à-dire la moyenne pondérée par les quantités, tous types confondus :

$\begin{array}{llll} & <\text { Temps total }>(\mathrm{h}) & <\text { Temps d'attente }>(\mathrm{h}) & \text { REP (\%) } \\ \text { Discipline FIFO } & 1304,53 & 697,93 & 46,49 \% \\ \text { Discipline AHP } & 961,35 & 354,32 & 63,14 \% \\ \text { Variation (\%) } & -26,31 \% & -49,23 \% & +35,81 \%\end{array}$

Tableau 3. Améliorations de performance obtenues.

Sur l'ensemble de la production, nous observons une diminution supérieure à $25 \%$ du temps moyen de production. Cette amélioration est totalement due à la réduction très forte des temps d'attente, de l'ordre de $50 \%$ en moyenne, soit une progression du REP de $35 \%$.

\section{Perspectives}

Le travail présenté ici représente les prémisses d'un nouvel axe de recherche en pilotage. De nombreuses améliorations, tant sur le plan fonctionnel, avec la prise en compte de nouveaux critères relatifs aux HP, HR et HO, que sur le plan technologique, avec la mise en œuvre d'un démonstrateur utilisant la simulation distribuée. 


\subsection{Prise en compte de nouveaux critères}

De nouveaux intérêts des différents holons peuvent être pris en considération. Nous évoquerons ici deux idées concernant principalement le holon ordre. Tout d'abord, nous pouvons travailler sur le regroupement et le fractionnement dynamiques de lots. Pour bénéficier des avantages de la production par lots sans en subir les inconvénients, il s'agit de conduire les transferts de produits entre ressources un par un, mais de regrouper les produits par références au sein de leurs encours respectifs. Lorsque le traitement d'un HP est achevé sur un HR, le I_ordre est décrémenté de 1 et le I_ordre correspondant aux HR suivant est incrémenté d'autant. Ceci correspond à une généralisation de la notion de chevauchement de lots. Une autre idée concerne la production de produits liés par une nomenclature d'assemblage. Produire une partie des produits nécessaires ne sert à rien tant que leur totalité n'est pas atteinte. Aussi, nous pouvons envisager un critère visant à assurer la synchronisation des livraisons de produits liés et la fiabilisation des dates de livraison à l'unité d'assemblage en aval de l'Unité 4-5. Ce critère peut être mis en œuvre via des informations attachées aux HO concernés par cette nomenclature.

\subsection{Utilisation de la simulation distribuée}

Dans le cadre d'autres travaux sur le pilotage holonique et isoarchique, nous avons développé en Java une plateforme de simulation distribuée utilisant la norme HLA (High Level Architecture) (IEEE P1516) (Ounnar et al., 2008). Nous avons utilisé cette technologie pour élaborer une seconde maquette, plus proche du système final et des concepts liés à PROSIS. Cela vise à obtenir un outil de pilotage interopérable, permettant de basculer aisément entre les 4 modes d'expérimentation (Pfeiffer et al., 2003) : le système de pilotage peut être étudié et/ou utilisé sur une partie opérative réelle ou émulée via un simulateur industriel.

\subsubsection{Simulation distribuée d'une fédération d'EPA}

Cette maquette permet de poursuivre les expérimentations dans des conditions proches du système réel de production (Figure 10). Dans cette plateforme, une holarchie de ressources est une fédération de simulation et chaque fédéré EPA est attaché à un holon ressource et supporte la I_ressource. Il accueille les I_ordres et I_produits nécessaires à l'expression de la circulation des holons ordres et produits. Il met en œuvre tous les mécanismēs de pilotage multicritère précédemment évoqués

Nous pourrons alors comparer les différentes performances obtenues par le système de production en configurant différents jeux de paramètres de l'algorithme AHP, et en cherchant à optimiser cette configuration. Nous obtenons alors une architecture générique de simulation pour un système de pilotage holonique isoarchique qui pourra être utilisé pour d'autres Job Shop.

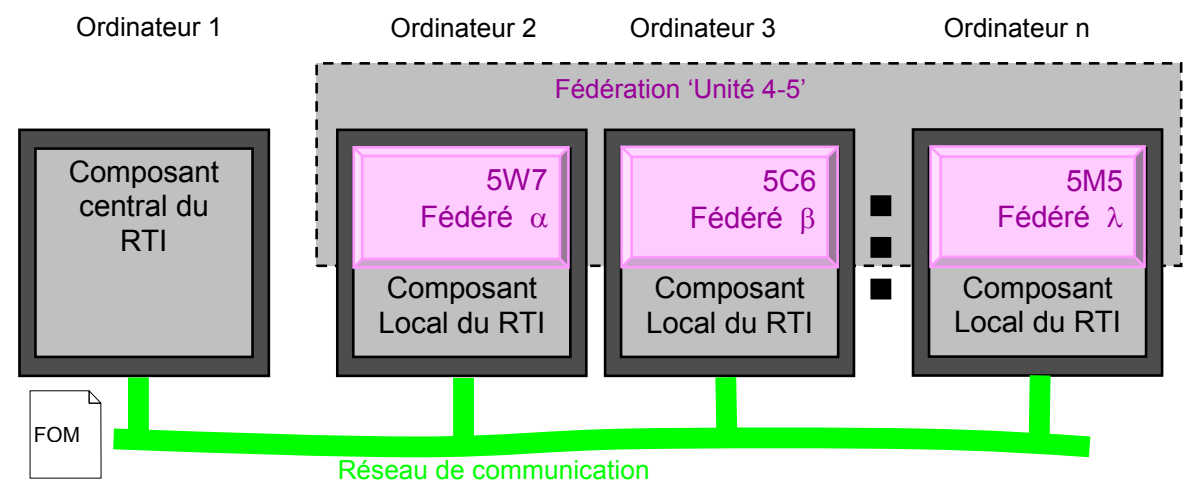

Figure 10. Structure de simulation HLA

Cette maquette pourra être également interopérable avec un atelier réel (c'est l'une des propriétés les plus intéressantes d'HLA...) : il pourra alors être mis en place des tests partiels n'impliquant qu'une partie d'un système de production.

Sur cette maquette, il est tout à fait possible de simuler un atelier de la taille de 1'Unité 4-5, mais il n'y a pas à priori de limitation de taille.

\subsubsection{Interface utilisateur d'une EPA}

A titre illustratif, la figure 11 montre une copie d'écran de l'IHM d'un poste de travail attaché à une EPA, permettant l'accès à toutes les données des holons concernés et à tous les paramètres des services proposés (AHP, indicateurs...). La partie droite de l'écran contient toutes les informations relatives à l'encours au pied de la machine. La partie gauche supérieure permet la visualisation de l'état de la ressource (machine, poste...). La partie gauche inférieure concerne les échanges d'informations liées à la circulation en parallèle des produits entre les ressources. 


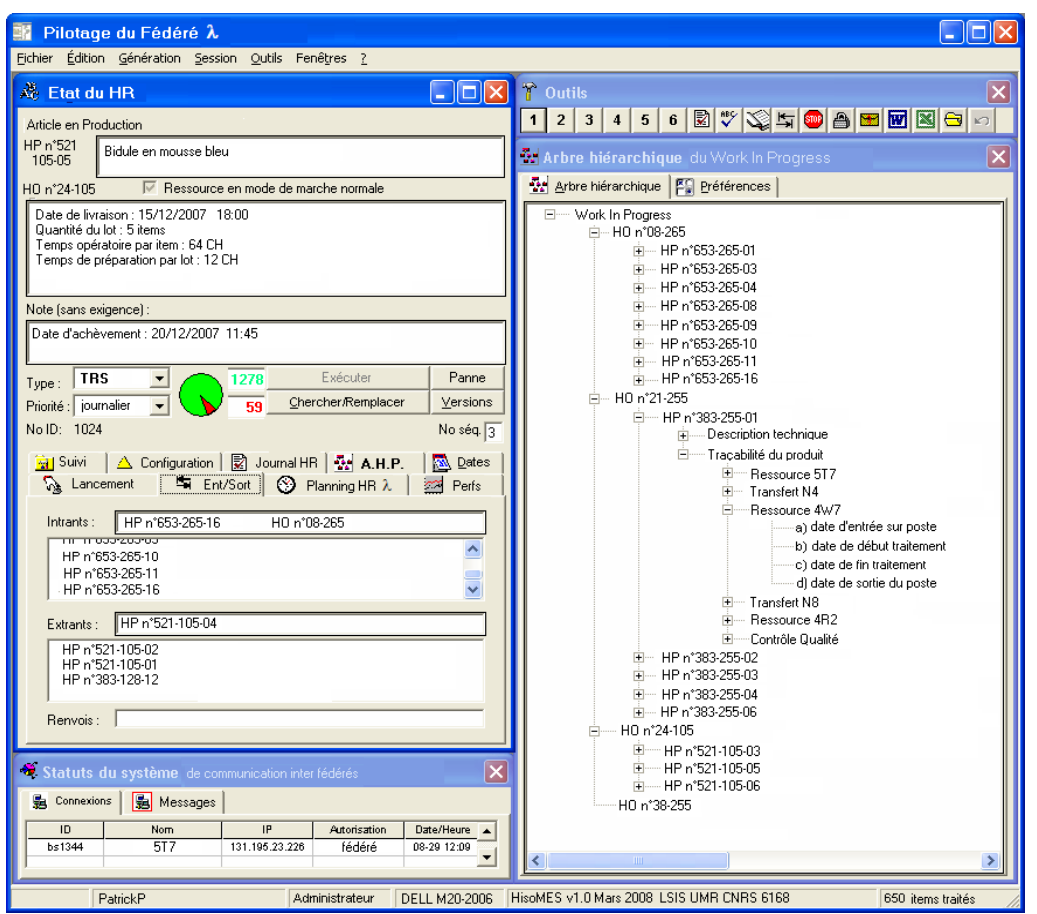

Figure 11. IHM d'un poste de travail

\subsubsection{Emulation des $M$ holons via Arena}

Avant de connecter le système de pilotage holonique et isoarchique sur un atelier réel, il est nécessaire d'en valider le fonctionnement. Pour cela, nous avons adopté le concept d'émulation proposé par (Castagna et al., 2008), en connectant la fédération d'EPA sur un modèle de simulation concernant les $M$ holons ( $M$ ressource, $M$ _produit et $M \_$ordre). Pour réaliser cela, plutôt que d'utiliser une solution à base de modèles GDEVS (Pujo et $\overline{a l}$., 2008), approche formelle du point de vue de la simulation, mais complexe à mettre en œuvre, nous avons choisi d'utiliser des modèles Arena directement pilotés par une communication TCP/IP. Chaque I_ressource est ainsi en relation via l'EPA avec le modèle de la M_ressource.

Au sein d'un modèle Arena, les entités modélisent les M_produits. Chacune de ces entités est identifiable de manière unique, cet identificateur permet ainsi de lier I_produit et M_produit.

Les $M_{-}$ressources sont modélisées par des blocs provenant de la bibliothèque que nous avons développés. La figure 12 présente le modèle logique et le modèle représentatif des M_ressources au sein d'Arena.

Une M_ressource est constituée d'une file d'attente d'entrée, d'une ressource et de sa propre file d'attente. La file d'attente d'entrée n'est pas liée à la ressource au sein du modèle logique. Cette file d'attente est utilisée comme stock tampon, pour classer, ordonner et éventuellement constituer des lots de M_produits. Un fois ces opérations d'organisation et d'agencement réalisées, les M_produits ou lots de $M$ _produits sont envoyés dans la file d'attente de la ressource, puis transformés par celle-ci, en fonction des classements successifs établis au sein de l'EPA.

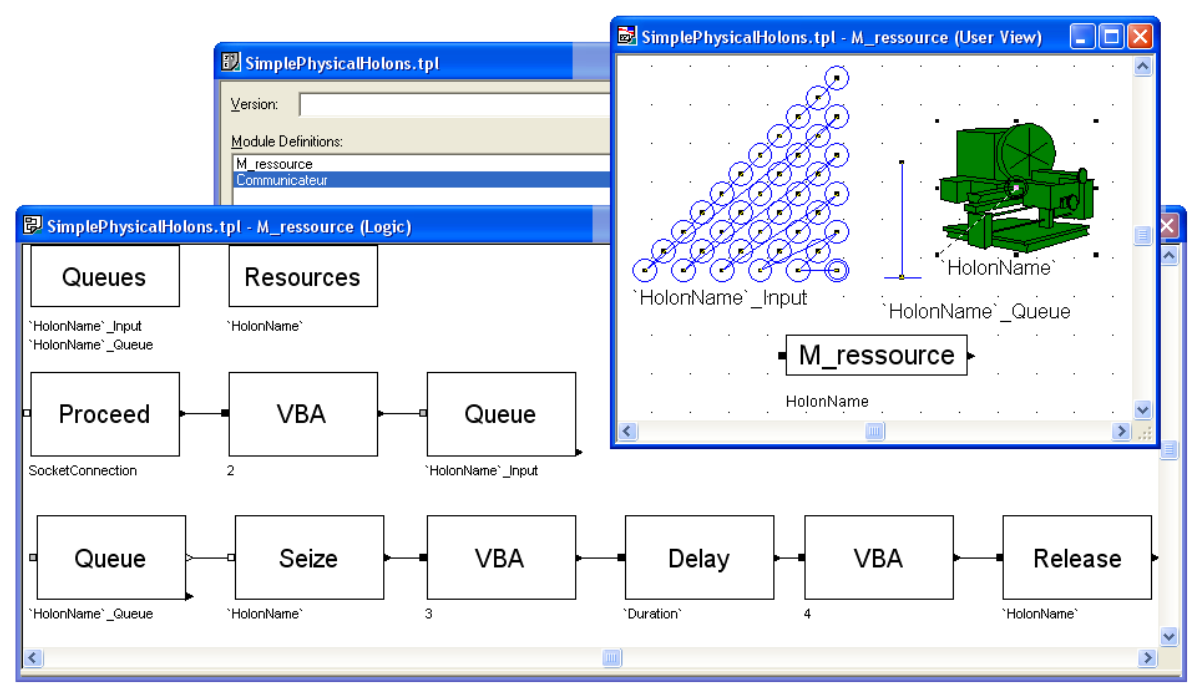

Figure 12. Modèle d'émulation sous Arena des M_ressources 
La synchronisation entre les I_produits (informations concernant les produits) et les M_produits (produits physiques ou émulés) est réalisée par l'EPA à des points situés au sein des M_ressources. Cette synchronisation permet ainsi d'actualiser les informations contenues dans l'EPA et les évènements qui ont lieu au sein de la structure matérielle (réelle ou émulée). Par exemple, l'arrivée d'un $M \_$produit dans un buffer d'attente, peut être signalé à l'EPA, afin de faire passer le I_produit correspondant dans le WIP et de le prendre en compte dans le classement AHP.

Dans une structure matérielle réelle, cette synchronisation peut être réalisée en utilisant la technologie RFID, la lecture des tags associés aux M_produits se faisant par le coupleur RFID de l'EPA. Les M_ressources émulées proposent le même principe de synchronisation, à trois points différents qui correspondent à des emplacements possibles de coupleur : à l'arrivée des M_produits dans la M_ressource (buffer d'attente), quand une opération est réalisée sur un M_produit, et à la fin de cette même opération.

Dans le cas des M ressources émulées, la lecture des tags RFID est modélisée par l'envoi d'un message au format XML. Ce message est envoyé à l'EPA par l'intermédiaire d'une socket utilisant le protocole TCP/IP et contenue dans un objet spécifique de la bibliothèque développée : le Communicateur.

Chaque synchronisation réalisée au niveau des blocs VBA du modèle logique des M_ressources, suspend la simulation (l'émulation) du système opérant. Un dialogue est établi entre l'EPA et le modèle émulé. Les décisions prisent au niveau de l'EPA sont alors transmises à l'émulateur, la simulation ne reprend ensuite que lors de la réception d'une décision de reprise en provenance de l'EPA.

Le développement d'une bibliothèque d'objets Arena présente l'avantage de permettre d'encapsuler les objets nécessaires au fonctionnement du modèle émulé (M_ressource et Communicateur), et d'utiliser ensuite ces objets dans des modèles Arena simulant le fonctionnement de M_ressources ou d'autres ressources ou files d'attentes.

La figure 13 présente un modèle pour lequel trois $M_{-}$ressources sont émulées et ou tout le système de transitique est simulé indépendamment des EPA, directement par Arena. Par _ ailleurs, la fenêtre en bas à gauche de cette figure montre les logs de la communication entre le système opérant émulé sous Arena des M_ressources et la fédération des EPA.

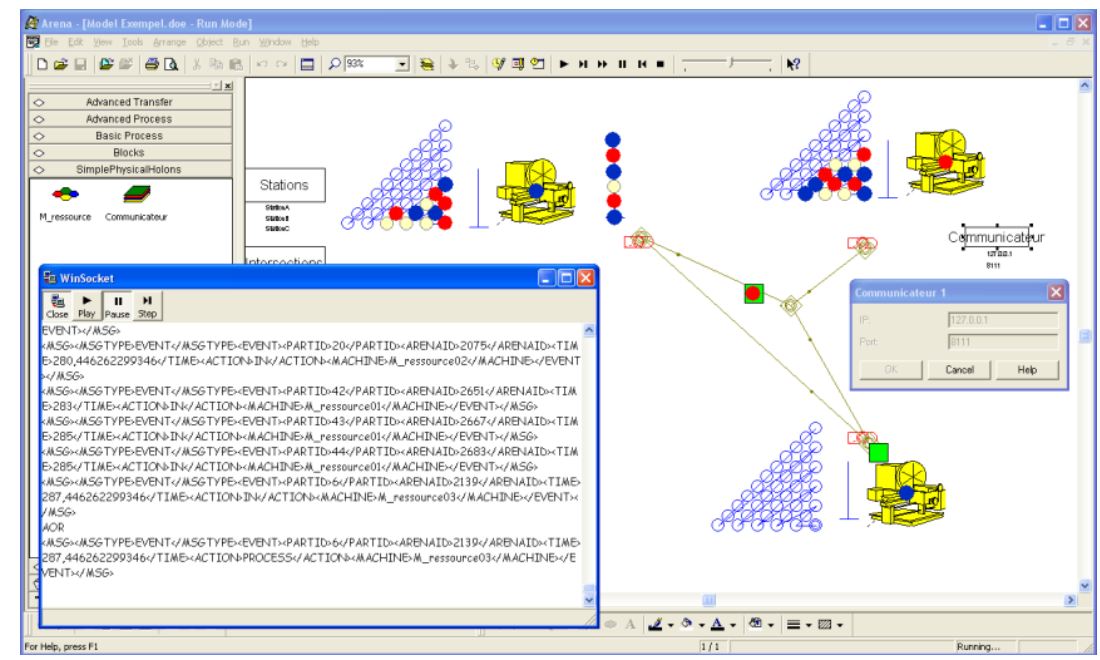

Figure 13. Modèle d'émulation du système opérant sous Arena

\section{Conclusion}

Nous avons proposé une architecture de pilotage auto organisé s'appuyant sur une approche holonique et isoarchique intitulée PROSIS. Le mode de décision multicritère via AHP actuellement testé dans PROSIS permet à chaque Holon Ressource, par l'intermédiaire de son EPA, de choisir en temps réel parmi les Holons Produits en attente celui dont le traitement favorise l'écoulement des flux, donc la diminution des durées de production, des volumes d'encours et des coûts associés, et cela sans faire référence à un quelconque ordonnancement prévisionnel. Nous avons présenté le déroulement de l'algorithme AHP mis en œuvre par l'EPA et les critères et indicateurs actuellement testés.

Les premiers résultats obtenus sur un cas industriel complexe sont encourageants : même s’ils sont sûrement encore améliorables, ils montrent sans équivoque l'intérêt d'un pilotage auto organisé et multicritères par rapport aux approches traditionnelles.

Par ailleurs, nous souhaitons tester de nouveaux critères : les systèmes de pilotage isoarchiques sont prometteurs, mais restent à explorer plus largement. Le plus difficile reste de définir les bonnes règles de fonctionnement qui permettent d'obtenir la performance recherchée vis-à-vis d'objectifs à atteindre et de critères d'évaluation. Grâce à la plateforme de simulation dont nous disposons maintenant, l'exploration des possibilités offertes par PROSIS est ouverte. 


\section{Références}

Bajic E. Y. Sallez, 2007. Proposition de projet transversal exploratoire du GDR MACS : «Système Contrôlé par le Produit », Journées prospectives STP, Lyon, 11 \& 12 juin.

Balasubramanian S., Brennan R. W., Norrie D. H., 2001. An architecture for metamorphic control of holonic manufacturing systems, Computers in Industry, 46, p.13-31.

Bongaerts L., L. Monostori, D. McFarlane, B. Kadar, 2000. Hierarchy in distributed shop floor control, Computers In Industry, 43, p. 123-137.

Bourrières J.P., P. Baptiste, A. Bernard, P. Lopez, G. Morel, H. Pierreval, M.C. Portmann, 2007. Comité d'Experts Productique : prospective de recherche, 15 janvier 2007.

Broissin N., 1999. Contribution a l'amélioration de la réactivité des systèmes de production automatises et flexibles grâce a un pilotage base sur une génération de taches décentralisée, Thèse de Doctorat en Sciences, Université d'Aix-Marseille III

Castagna P., A. Thomas, P. Blanc, O. Cardin, R. Pannequin, H. El Haouzi, T. Klein, 2008. La simulation pour les systèmes pilotés par le Produit, dans : Simulation pour le pilotage de la chaîne logistique, Hermès Sciences

Charpentier P., F. Chaxel, A. Thomas, E. Muhl, 2001. Sur quelques approches de pilotage distribué et leur formalisation, JESA, 35 (7-8), p.885-904

Chaxel F., E. Bajic, J. Richard, 1999. Automotive Vehicules Data Management Based on the Product Holon Paradigm, IEEE Systems Man \& Cybernetics, Tokyo, Japon, 12-15 Octobre 1999.

Deen, S.M., 2003. Agent-Based Manufacturing - Advances in the Holonic Approach, Springer-Verlag Ed, ISBN 3-540-44069-0.

Gouyon D., 2004. Contrôle par le produit des systèmes d'exécution de la production : apport des techniques de synthèse. Thèse de Doctorat, Université Henry Poincaré, Nancy.

IEEE P1516-2, 2000. Draft Standard for Modeling and Simulation (M\&S) High Level Architecture (HLA) - Framework and Rules, IEEE Standard for Modeling and Simulation.

Koestler A., 1967. The Ghost in the Machine, Hutchinson, London.

Leitao P., Restivo F., 2006. ADACOR: A holonic architecture for agile and adaptive manufacturing control, Computers in Industry 57, p. 121-130

Mathews J. 1995. Organizational foundations of intelligent manufacturing systems - the holonic viewpoint. Computer Integrated Manufacturing Systems, 8(4), p. 237-243.

McFarlane D., S. Sarla, J. Chirn, C. Wong, K. Ashton. 2002. The intelligent product in manufacturing control, Journal of EAIA.

Mekaouche L., 2007. Pilotage holonique auto-organisé de réseaux logistiques : validation par modélisation et simulation distribuée. Thèse de Doctorat, Université Paul Cézanne - Marseille.

Mesarović M. D., D. Macko, Y. Takahara, 1980. Théorie des systèmes hiérarchiques à niveaux multiples, Economica, Paris.

Morel G., P.Valckenaers, J.-M. Faure, C. Pereira, C. Dietrich, 2007. Manufacturing plan control challenges and issues, Control Engineering Practice, 15 (11), p.1321-1331.

Ounnar F., 1999. Prise en compte des aspects décision dans la modélisation par réseaux de Petri des systèmes flexibles de production. Thèse de Doctorat, INPG - Grenoble.

Ounnar F., P. Pujo, L. Mekaouche, N. Giambiasi, 2007. Customer-supplier relation-ship management in an intelligent supply chain network. Production Planning \& Control, 18(5), p. 377--387

Ounnar F., Pujo P., Mekaouche L., Giambiasi N., 2008. Integration of a flat holonic form in an HLA environment, Journal of Intelligent Manufacturing, DOI: 10.1007/s10845-008-0106-4

Panetto H., S. Baïna, G. Morel, 2007. Mapping the IEC 62264 models onto the Zachman framework for analysing products information traceability: a case study, Journal of Intelligent Manufacturing 18 (6) 679-698

Pannequin R., A. Thomas, 2006. "Cooperation between business and holonic manufacturing decision systems ». $12^{\text {th }} I F A C$ Symposium on Information Control Problems in Manufacturing (INCOM'2006), mai, St Etienne, p. 423-428.

Pétin J-F., D. Gouyon, G. Morel, 2007. Supervisory synthesis for product-driven automation and its application to a flexible assembly cell, Control Engineering Practice , 15 (5), p. 595-614

Pfeiffer A., B. Kadar, L. Monostori, 2003. « Evaluating and Improving Production Control Systems by using Emulation », IC on Applied Simulation and Modelling, (ASM 2003), September 3-5, Marbella, Spain, pp.:261-267.

Pujo P., J.P. Kieffer, 2002. Concepts fondamentaux du pilotage des systèmes de production. Dans : Fondements du pilotage des systèmes de production, Traité IC2 Productique (Pujo P. and J.P. Kieffer. Ed), p. 25-50, Hermès, Paris.

Pujo P., D. Brun-Picard, 2002. Pilotage sans plan prévisionnel, ni ordonnancement préalable. Dans : Méthodes du pilotage des systèmes de production, Traité IC2 Productique (Pujo P. et J.P. Kieffer. Ed), p. 129-162, Hermès, Paris.

Pujo P., M. Pedetti, F. Ounnar, 2004. Pilotage proactif des lignes de production kanban par modélisation DEVS et simulation temps réel, MOSIM'04, Nantes, France, 1-3 septembre.

Pujo P., F. Ounnar, 2007. Vers une approche holonique des systèmes mécatroniques complexes - Proposition d'un système de pilotage auto-organisé et isoarchique, JESA, 41 (6), p. 673-706 - doi:10.3166/jesa.41.673-706

Pujo P., G. Zacharewicz, C. Frydman, N. Giambiasi, 2008. Pilotage assisté par simulation distribuée dans l'industrie du semi conducteur, MOSIM'08, Paris.

Saaty T., 1980. The Analytic Hierarchy Process, Mc Hill.

Trentesaux D., 2002. Pilotage hétérarchique des systèmes de production, Mémoire d'Habilitation à Diriger des Recherches, Université de Valenciennes et du Hainaut Cambrésis.

Trentesaux D., 2007. Les systèmes de pilotage hétérarchiques, JESA, 41 (9-10), p.1165-1202 - doi:10.3166/jesa.41.1165-1202

Van Brussel H., J. Wyns, P. Valkenaers, L. Bongaerts, P. Peeters, 1998. Reference architecture for holonic manufacturing systems: PROSA, Computers in Industry, 37, p. 255-274. 